\title{
Head and Neck Oncogenesis - An Evolving Conundrum with Molecular Shades
}

\section{Rui Amaral Mendes*}

Professor of Oral Medicine, Oral Oncology and Oral Surgery, Institute of Health Sciences, The Catholic University of Portugal, Portugal

Histologically, the progression towards malignancy includes sequential pathological alterations ranging from hyperplasia through dysplasia to carcinoma in situ and invasive carcinoma and is determined by the accumulation of a series of genetic events.

Carcinogenic events lead to a molecular and histological multistage process featuring genetic and phenotypic markers for each stage, which involves enhanced function of several oncogenes and/or the deactivation of tumor suppressor genes, resulting in the loss of cell cycle checkpoints.

In cellular carcinogenesis various genes interact with each other, thus leading to multiple alterations that occur in a rather complex way and in different stages of progression of the disease.

Focus on pre-invasive stages of high-grade and low-grade lesions that are known to further develop into cancer have allow us to identify early genetic events during cancer development, as genetic alterations build up a neoplastic phenotype.

On the other hand, research involving carcinogenesis of the Head and Neck over the last decade revealed that a panel of genes may help to separate normal oral epithelia from cancerous one, further highlighting the importance of epigenetic silencing.

Epigenetic alterations, which comprise DNA methylation, histone modifications, nucleosome positioning, and small, noncoding RNAs ( $m i R N A, s i R N A$ ), are involved in transcriptional changes and decisive events that will determine cell fate and phenotype and are widely described as essential players in cancer progression [1].

Still, although miRNA profile changes in the outline of dysplastic cells assent their molecular fingerprint, results on cancer cell lines require further analyses to understand whether altered miRNA levels are a consequence or rather a cause of the changes reported in these proteins.

Moreover, discordant results among cancers/cell lines of different part of the mouth, may confirm biological differences, which may lead to different diagnostic/therapeutic pathways.

Likewise, changes in cell morphology, proliferation, longevity and survival, as well as angiogenesis and other properties that characterize cancer cells are often produced by alterations in several signal transduction pathways.

Hence, comprehension of the underlying pathways governing the progression of oral potential malignant disorders or lesions is of the utmost importance. The molecular changes antedate the occurrence of invasive malignancy and can be used for risk assessment and as intermediate endpoints for targeted therapies.

In fact, several pathways have been reported to be alternate in Head and Neck carcinogenesis (e.g. Receptor Tyrosine Kinase, FGF, PI3K/AKT, COX-2, NFkB, ERK/MAPK, Cyclin D1, VEGF and Wnt pathways, as well as cell adhesion molecules).
The FGF signaling pathway, an important therapeutic target in human cancers, not only regulates the developmental processes and angiogenesis, but it also activates the PI3K/AKT signaling cascade, leading to an induction of the important epithelial-mesenchymal transition and to cell migration [2].

The CCND1 gene, located at 11q13, encodes protein cyclin D1 which is a key regulator of the G1 phase of the cell cycle. A recent pathway-based approach to assess the complex interaction between Single-Nucleotide Polymorphisms (SNPs) from genes in the cellcycle control pathway and the overall risk of oral premalignant lesions showed that CCND1 P241P polymorphism was significantly associated with an increased risk of oral premalignant lesions for the variant homozygous genotype. Compared with the common homozygous genotype (GG), the rare homozygous genotype (AA) of CCND1 was associated with a 2.5-fold increased risk of oral premalignant lesion [3]. Another case-control study of 115 oral premalignant lesions and 230 controls showed that individuals with one or more copies of the CCND1 G870A variant A-allele present an increased risk of oral premalignant lesion development, thus concluding that the results supported the hypothesis that this polymorphism could be a susceptibility factor in the molecular progression of oral cancer [4].

Interestingly, assessment of gene amplifications occurring in oral dysplasia and, most importantly, oncogenic pathways disrupted in premalignancies showed that gene amplification did occur in $40 \%$ of the early lesions but, most importantly, that affected genes were part of different canonical oncogenic pathways, of which some shared common nodes and interact as a single network [2].

Consequently, instead of the concept of "oncogene addiction", which tends to call for a physiological dependence of cancer cells on a single activated oncogene, the fact that some cases exhibited multiple amplicons harboring genes from the same signaling pathways are suggestive of what some authors designate a "pathway addiction" pattern [2].

Overall, engaging a particular molecular event may improve the currently available diagnostic tools and further lead to a more tailoredoriented clinical management of oral neoplastic and pre-neoplastic lesions.

*Corresponding author: Rui Amaral Mendes, Professor of Oral Medicine, Oral Oncology and Oral Surgery, Institute of Health Sciences, The Catholic University of Portugal, Portugal, Tel: + 351-93-2805962; E-mail: ramaralmendes@crb.ucp.pt

Received October 22, 2013; Accepted October 24, 2013; Published October 29 2013

Citation: Mendes RA(2013) Head and Neck Oncogenesis - An Evolving Conundrum with Molecular Shades. J Carcinog Mutagen S5: e001. doi:10.4172/2157-2518.S5 e001

Copyright: (C) 2013 Mendes RA. This is an open-access article distributed under the terms of the Creative Commons Attribution License, which permits unrestricted use, distribution, and reproduction in any medium, provided the original author and source are credited. 
Citation: Mendes RA (2013) Head and Neck Oncogenesis - An Evolving Conundrum with Molecular Shades. J Carcinog Mutagen S5: e001. doi:10.4172/2157-2518.S5-e001

Page 2 of 2

\section{References}

1. Gasche JA, Goel A (2012) Epigenetic mechanisms in oral carcinogenesis Future Oncol 8: 1407-1425.

2. Tsui IF, Poh CF, Garnis C, Rosin MP, Zhang L, et al. (2009) Multiple pathways in the FGF signaling network are frequently deregulated by gene amplification in oral dysplasias. Int J Cancer 125: 2219-2228.
3. Ye Y, Lippman SM, Lee JJ, Chen M, Frazier ML, et al. (2008) Genetic variations in cell-cycle pathway and the risk of oral premalignant lesions. Cancer 113 2488-2495.

4. Huang M, Spitz MR, Gu J, Lee JJ, Lin J, et al. (2006) Cyclin D1 gene polymorphism as a risk factor for oral premalignant lesions. Carcinogenesis 27: 2034-2037.
This article was originally published in a special issue, Oncogenomics of the Head and Neck handled by Editor(s). Dr. Rui Amaral Mendes, Portuguese Catholic University, Portugal 